\title{
REFINEMENT MONOIDS WITH WEAK COMPARABILITY AND APPLICATIONS TO REGULAR RINGS AND $C^{*}$-ALGEBRAS
}

\author{
P. ARA AND E. PARDO
}

(Communicated by Ken Goodearl)

Dedicat al petit Guillem

\begin{abstract}
We prove a cancellation theorem for simple refinement monoids satisfying the weak comparability condition, first introduced by K.C. O'Meara in the context of von Neumann regular rings. This result is then applied to von Neumann regular rings and $C^{*}$-algebras of real rank zero via the monoid of isomorphism classes of finitely generated projective modules.
\end{abstract}

\section{INTRODUCTION}

Let $M$ be an (abelian) monoid. For $x, y \in M$ we will write $x \leq y$ if there exists $z \in M$ such that $y=x+z$. This preorder is sometimes called the algebraic preorder in $M$. Let $M^{*}$ denote the set of nonzero elements of $M . M$ is called conical if $M^{*}$ is closed under addition. We will write $x \leq^{*} y$ if there exists $z \in M^{*}$ such that $y=x+z$. Note that the relation $\leq^{*}$ is transitive if $M$ is a conical monoid. An order-unit in $M$ is a nonzero element $u \in M$ such that for each $x \in M$ there exists $n \geq 1$ such that $x \leq n u$. A monoid $M$ is said to be simple if it is nonzero and every nonzero element of $M$ is an order-unit, so that $M$ has no nontrivial ideals (i.e. convex submonoids of $M$ ).

Definition. Let $(M, u)$ be a monoid with order-unit. We say that $(M, u)$ satisfies weak comparability provided that for all nonzero elements $x$ in $M$ such that $x \leq u$, there exists a positive integer $k=k(x)$ such that, if $y \in M$ and $k y \leq u$, then $y \leq x$. Note that, for conical $M$, if there is an element $x^{\prime} \in M^{*}$ such that $x^{\prime} \leq^{*} x$, then replacing $k(x)$ by $k\left(x^{\prime}\right)$ we obtain a positive integer $k$ such that $k y \leq u$ implies $y \leq * x$.

We say that a subset $X$ of a monoid $M$ is cancellative (respectively strictly cancellative) if, for $a, b, c \in X$, the relation $a+c=b+c$ (resp. $\left.a+c \leq^{*} b+c\right)$ implies $a=b$ (resp. $a \leq^{*} b$ ).

Our main results are the following:

Received by the editors May 19, 1994 and, in revised form, September 21, 1994.

1991 Mathematics Subject Classification. Primary 16E20, 16E50, 46L80, 19K14, 06F20.

Key words and phrases. Refinement monoid, $C^{*}$-algebra with real rank zero, von Neumann regular ring, weak comparability.

The research of the authors was supported by grants from the DGICYT (Spain). 
Theorem. Let $(M, u)$ be a simple refinement monoid satisfying weak comparability. Then $M^{*}$ is cancellative.

Corollary. Let $(M, u)$ be a simple refinement monoid with weak comparability. Assume that $u+a=u$ implies $a=0$ for all $a \in M$. Then $M$ is cancellative.

We can apply these results to obtain cancellation results for rings $R$ via the monoids of finitely generated projective right $R$-modules $V(R)$ provided we know that $V(R)$ is a refinement monoid. This is the case for von Neumann regular rings and for $C^{*}$-algebras with real rank zero. For von Neumann regular rings we recover O'Meara's Theorem [8, Theorem 1]. Also our methods prove that strict cancellation implies cancellation for $C^{*}$-algebras of real rank zero, giving a partial answer to $[\mathbf{3}$, Question 3.2.3].

\section{REFINEMENT MONOIDS}

A monoid $M$ is said to be a refinement monoid $[\mathbf{1 1}]$ if whenever $a+b=c+d$ in $M$, there exists $x, y, z, t \in M$ such that $a=x+y, b=z+t$ while $c=x+z$ and $d=y+t$. We say that $M$ satisfies the Riesz decomposition property if whenever $p \leq q_{1}+q_{2}$ in $M$, there exist $p_{1}, p_{2} \in M$ such that $p=p_{1}+p_{2}$ and $p_{i} \leq q_{i}$ for $i=1,2$. Clearly every refinement monoid satisfies the Riesz decomposition property, but the converse is not true in general. For example, the monoid $\{0, u, \infty\}$ (where $2 u=\infty$ ) satisfies the Riesz decomposition property but it is not a refinement monoid.

An atom of a monoid $M$ is a nonzero element $a$ such that there is no $b$ in $M$ such that $0 \leq^{*} b \leq^{*} a$. $M$ is an atomic monoid if each element of $M$ can be written as a sum of atoms. Clearly a simple, nonatomic, refinement monoid has no atoms. The following lemma is known. In fact, much more is true; see [10, Theorem 6.2].

Lemma 1.1. Let $M$ be a simple nonatomic refinement monoid and let $x_{1}, \cdots, x_{n} \in$ $M^{*}$. Then for each $k \in \mathbb{N}$ there exists $x \in M^{*}$ such that $k x \leq x_{i}$ for all $i$.

Lemma 1.2. Let $M$ be a simple, conical, nonatomic, refinement monoid and assume that $x \leq^{*} y+w$ for some $x, y, w \in M$ with $y \neq 0$. Then there exists $y^{\prime} \in M^{*}$ such that $x \leq^{*} y^{\prime}+w$ and $y^{\prime} \leq^{*} y$.

Proof. Write $x+t=y+w$ for some $t \in M^{*}$. Applying the refinement property, we have $x=x_{1}+x_{2}$ and $t=t_{1}+t_{2}$ such that $y=x_{1}+t_{1}$ and $w=x_{2}+t_{2}$. If $t_{1} \neq 0$, write $t_{1}=t_{1}^{\prime}+t_{1}^{\prime \prime}$ with $t_{1}^{\prime}, t_{1}^{\prime \prime} \in M^{*}$ and set $y^{\prime}=x_{1}+t_{1}^{\prime \prime}$. Then clearly $x \leq^{*} y^{\prime}+w$. If $t_{1}=0$, then, by Lemma 1.1, we can write $t_{2}=2 t_{2}^{\prime}+t_{2}^{\prime \prime}$ with $t_{2}^{\prime} \in M^{*}$ and $t_{2}^{\prime} \leq^{*} y$. Write $y=t_{2}^{\prime}+y^{\prime}$ for some $y^{\prime} \in M^{*}$. Then

$$
x \leq^{*} x+t_{2}^{\prime}+t_{2}^{\prime \prime}=y+t_{2}^{\prime}+t_{2}^{\prime \prime}+x_{2}=y^{\prime}+2 t_{2}^{\prime}+t_{2}^{\prime \prime}+x_{2}=y^{\prime}+w,
$$

as desired.

Lemma 1.3. If $(M, u)$ is a simple, conical, nonatomic, refinement monoid satisfying weak comparability, then $M^{*}$ is strictly cancellative.

Proof. Assume $x, y, z$ are elements of $M^{*}$ such that $x+z \leq^{*} y+z$. There exists $m \in \mathbb{N}$ such that $z \leq m u$, whence $x+m u \leq^{*} y+m u$. Since $M$ is conical, it suffices to prove the result for the case $z=u$.

So, assume that $x+u \leq^{*} y+u$ with $y \in M^{*}$. By Lemma 1.2, there exist $y^{\prime}, t \in M^{*}$ such that $y=y^{\prime}+t$ and $x+u \leq^{*} y^{\prime}+u$. By weak comparability, there 
is $k \in \mathbb{N}$ such that, for $a \in M$, if $k a \leq u$, then $a \leq^{*} t$. Now, using [11, Lemma 1.11(i)] we get decompositions $x=x_{1}+x_{2}$ and $y^{\prime}=x_{1}+y_{1}$ such that $k x_{2} \leq u$. Therefore, we conclude that $x_{2} \leq^{*} t$ and so $x=x_{1}+x_{2} \leq^{*} x_{1}+y_{1}+t=y^{\prime}+t=y$. It follows that $M^{*}$ satisfies strict cancellation.

Proposition 1.4. Let $(M, u)$ be a simple, conical, nonatomic, refinement monoid satisfying weak comparability.

(a) Assume that $x+u=u$ implies $x=0$ for $x \in M$. Then $M$ is strictly cancellative.

(b) Assume that $a+u=u$ for some $a \in M^{*}$. Then $x \leq^{*} y$ for all $x, y \in M^{*}$ and $M^{*}$ is cancellative.

Proof. (a) Since $M^{*}$ is strictly cancellative by Lemma 1.3, it will suffice to prove that $x+z=z$ implies $x=0$ for all $x, z \in M$.

Assume that $x+z=z$ for some $x \in M^{*}$. Since $M$ is nonatomic, we then have $x^{\prime}+z \leq^{*} z$ for some $x^{\prime} \in M$. Now there exists a positive integer $n$ such that $z \leq n u$ and so $x^{\prime}+n u \leq^{*} n u$. By applying Lemma 1.3 we obtain $x^{\prime}+u \leq^{*} u$, giving a contradiction.

(b) Assume that $a+u=u$ for some $a \in M^{*}$. Let $x, y$ be elements in $M^{*}$. There exists $n \geq 1$ such that $x \leq n a$ and thus $x+u \leq n a+u=u$. We have $x+u \leq u \leq^{*} y+u$ and consequently Lemma 1.3 gives us $x \leq^{*} y$.

Now assume that $x+z=y+z$ for $x, y, z \in M^{*}$. Then $z \leq^{*} x$ and $z \leq^{*} y$ so that $x=z+x^{\prime}$ and $y=z+y^{\prime}$ for some $x^{\prime}, y^{\prime} \in M^{*}$. Now $2 z \leq^{*} z$ implies $z=2 z+t$ for some $t \in M^{*}$ and so

$$
x=z+x^{\prime}=2 z+t+x^{\prime}=x+z+t=y+z+t=2 z+y^{\prime}+t=z+y^{\prime}=y,
$$

showing cancellation in $M^{*}$.

Lemma 1.5. Let $M$ be a strictly cancellative, conical, atomless, refinement monoid Assume that $2 a+h=a+y$ for some $a, h, y \in M$. Then there exists $e \in M$ such that $2 a=a+e$ and $y=h+e$.

Proof. By applying the refinement property to the equality $a+a+h=a+y$, we get $a=a_{1}+t_{1}=a_{2}+t_{2}$ and $h=a_{3}+t_{3}$, with $a_{1}+a_{2}+a_{3}=a$ and $t_{1}+t_{2}+t_{3}=y$. If $a_{3}=0$, then we can take $e=t_{1}+t_{2}$. So, assume that $a_{3} \neq 0$. Since $M$ has no atoms, there exists nonzero $x_{1}, x_{2} \in M$ such that $a_{3}=x_{1}+x_{2}$.

For $i=1,2$, we have $a_{i}+x_{i} \leq^{*} a_{1}+a_{2}+x_{1}+x_{2}=a=a_{i}+t_{i}$. Since $M$ is strictly cancellative, we get $x_{i} \leq^{*} t_{i}$ for $i=1,2$. Write $t_{i}=x_{i}+x_{i}^{\prime}$ for some $x_{i}^{\prime} \in M^{*}$, and set $e=x_{1}^{\prime}+x_{2}^{\prime}$. Then we have

$$
y=t_{1}+t_{2}+t_{3}=x_{1}+x_{1}^{\prime}+x_{2}+x_{2}^{\prime}+t_{3}=a_{3}+t_{3}+e=h+e
$$

and also

$$
\begin{aligned}
& a+e=a_{1}+a_{2}+a_{3}+x_{1}^{\prime}+x_{2}^{\prime}=a_{1}+a_{2}+x_{1}+x_{2}+x_{1}^{\prime}+x_{2}^{\prime} \\
& =a_{1}+t_{1}+a_{2}+t_{2}=2 a .
\end{aligned}
$$

This completes the proof.

We need a simple lemma: 
Lemma 1.6. The only conical atomic simple refinement monoid is the infinite cyclic monoid.

Proof. Let $M$ be a conical atomic simple refinement monoid and let $a$ be an atom in $M$. By Riesz decomposition, every element $b$ in $M$ is of the form $n a$ for some nonnegative integer $n$. Assume there is a relation $n a=m a$ for $0 \leq n<m$. Then $n>0$ because $M$ is conical. Now use the refinement property to get decompositions $a=\sum_{j=1}^{m} a_{i j}$ for $i=1, \ldots, n$ such that $a=\sum_{i=1}^{n} a_{i j}$ for $j=1, \ldots, m$. Since $n<m$, there exists some $i$ such that the identity $a=\sum_{j=1}^{m} a_{i j}$ reads $a=k a$ for some $k>1$. But then $a \leq^{*} a$, contradicting the fact that $a$ is an atom.

Theorem 1.7. Let $(M, u)$ be a simple refinement monoid satisfying weak comparability. Then $M^{*}$ is cancellative.

Proof. If $M$ is not conical, then it is easy to show, by using the simplicity, that $M$ is a group. So, assume that $M$ is conical (so that $M^{*}$ is a semigroup). By Lemma 1.6 we can assume in addition that $M$ is nonatomic. By Proposition 1.4, we only need to consider the case in which $M$ is strictly cancellative.

So, let $M$ be a strictly cancellative, simple, conical, nonatomic, refinement monoid. Let $x, y, z \in M^{*}$ such that $x+z=y+z$. By Lemma 1.1, there exists $w \in M^{*}$ such that $2 w \leq x$. By simplicity there exists a positive integer $k$ such that $z \leq k w$. Using Riesz decomposition we get $z=z_{1}+\cdots+z_{k}$ such that $z_{i} \leq w$ for all $i$. So we get

$$
x+z_{1}+\cdots+z_{k}=y+z_{1}+\cdots+z_{k}
$$

with $2 z_{i} \leq x$ and we therefore can reduce the problem to the case in which $2 z \leq x$. So, assuming this condition, write $x=2 z+t$ for some $t \in M$ and note that $2 z+(t+z)=z+y$. By Lemma 1.5, there exists $e \in M$ such that $2 z=z+e$ and $y=t+z+e$. Therefore

$$
x=2 z+t=z+e+t=y,
$$

showing cancellation in $M^{*}$.

Corollary 1.8. Let $(M, u)$ be a simple refinement monoid with weak comparability. Assume that $u+a=u$ implies $a=0$ for all $a \in M$. Then $M$ is cancellative.

Proof. We can again assume that $M$ is conical. Since $M^{*}$ is cancellative by Theorem 1.7, it suffices to prove that $a+c=c$ implies $a=0$ for all $a, c \in M$. Since $M$ is simple, there exists $n \geq 1$ such that $c \leq n u$. So $a+n u=n u$ and Theorem 1.7 tells us that $a+u=u$. We conclude that $a=0$.

Finally we note that the proof of Theorem 1.7 gives the following:

Corollary 1.9. Let $M$ be a simple strictly cancellative refinement monoid. Then $M$ is cancellative.

The hypothesis of Corollary 1.9 is weaker than those in Corollary 1.8, because there exist simple cancellative refinement monoids which do not satisfy weak comparability; see [9, Example 3.12]. 


\section{Applications to CANCELlation \\ RESULTS FOR SOME RINGS AND $C^{*}$-ALGEBRAS}

Let $R$ be a ring and let $V(R)$ be the monoid of isomorphism classes of finitely generated projective right $R$-modules. It is well-known that $V(R)$ is a refinement monoid whenever $R$ is a von Neumann regular ring [6, Theorem 2.8]. Also, a von Neumann regular ring is unit-regular if and only if $V(R)$ is a cancellative monoid [6, Theorem 4.5]. So Corollary 1.8 gives immediately the following result, due to K.C. O'Meara.

Theorem 2.1 ([8, Theorem 1]). Let $R$ be a simple directly finite von Neumann regular ring satisfying weak comparability. Then $R$ is unit-regular.

Also we have the following consequence of Corollary 1.9:

Theorem 2.2. Let $R$ be a simple von Neumann regular ring such that $A \oplus C \prec$ $B \oplus C$ implies $A \prec B$ for all finitely generated projectives $A, B, C$. Then $R$ is unit-regular.

Now we turn our attention to $C^{*}$-algebras. We will follow the background and notation of $[\mathbf{2}]$ and $[\mathbf{3}]$. The monoid $V(A)$ can be identified with the monoid of Murray-von Neumann equivalence classes of projections in $M_{\infty}(A)$. For projections $p, q$ in a $C^{*}$-algebra, we write $p \lesssim q$ if $p$ is Murray-von Neumann equivalent to a subprojection of $q$. The notation $k \cdot p$ for a positive integer $k$ and a projection $p$ will mean the orthogonal sum of $k$ copies of $p$.

Definition. We say that a unital $C^{*}$-algebra $A$ satisfies weak comparability if for every nonzero projection $p$ in $A$ there exists a positive integer $k$ such that, for any projection $q$ in $A$, if $k \cdot q \lesssim 1$, then $q \lesssim p$.

It is clear that $A$ satisfies weak comparability if and only if $(V(A),[1])$ satisfies weak comparability.

Lemma 2.3. Let $A$ be a $C^{*}$-algebra with real rank zero. Then $V(A)$ is a refinement monoid.

Proof. By [5, Theorem 2.10], all the matrix algebras over $A$ have real rank 0. So it suffices to consider two projections $p, q$ in a unital $C^{*}$-algebra with real rank zero and show decompositions $q=q_{11}+q_{12}$ and $1-q=q_{21}+q_{22}$ such that $q_{11}+q_{21} \sim p$ and $q_{12}+q_{22} \sim 1-p$.

By [12, Theorem 3.2], $q$ is homotopic to a projection of the form $p^{\prime}+p^{\prime \prime}$ where $p^{\prime} \leq p$ and $p^{\prime \prime} \leq 1-p$. So we can write $q=q_{11}+q_{12}$ where $q_{11} \sim p^{\prime}$ and $q_{12} \sim p^{\prime \prime}$. Since $1-q$ is homotopic to $1-\left(p^{\prime}+p^{\prime \prime}\right)=\left(p-p^{\prime}\right)+\left(1-p-p^{\prime \prime}\right)$, we obtain a corresponding decomposition $1-q=q_{21}+q_{22}$. Finally observe that $q_{11}+q_{21} \sim p^{\prime}+\left(p-p^{\prime}\right)=p$ and $q_{12}+q_{22} \sim p^{\prime \prime}+\left(1-p-p^{\prime \prime}\right)=1-p$, as desired. $\square$

Theorem 2.4. Let $A$ be a finite simple unital $C^{*}$-algebra with real rank zero satisfying weak comparability. Then $V(A)$ has cancellation, the stable rank of $A$ is one, and $K_{0}(A)$ is a weakly unperforated Riesz group.

Proof. By Lemma 2.3, $V(A)$ is a refinement monoid. So, $V(A)$ is cancellative by Corollary 1.8. By [4, III.2.4], the stable rank of $A$ is one. Finally, by $[\mathbf{1}$, Theorem $4.2], K_{0}(A)$ is weakly unperforated.

The following result answers [3, Question 3.2.3] for $C^{*}$-algebras with real rank zero. 
Theorem 2.5. Let $A$ be a simple $C^{*}$-algebra with real rank zero such that $V(A)$ is strictly cancellative. Then the stable rank of $A$ is one.

Proof. It follows immediately from Lemma 2.3, Corollary 1.9 and [4, III.2.4].

\section{ACKNOWLEDGMENT}

It is a pleasure to thank Ken Goodearl for his useful suggestions.

\section{REFERENCES}

1. P. Ara, K.R. Goodearl, E. Pardo and D. Tyukavkin, K-Theoretically simple von Neumann regular rings, Journal of Algebra 174 (1995), 659-677.

2. B. Blackadar, K-Theory for operator algebras, MSRI Publications 5, Springer-Verlag, New York, 1986. MR 88g:46082

3. B. Blackadar, Comparison theory for simple $C^{*}$-algebras, in "Operator algebras and application", D.E. Evans and M. Takesaki (eds.), LMS Lecture Notes Series 135, Cambridge Univ. Press, 1988, pp. 21-54. MR 90g:46078

4. B. Blackadar and D. Handelman, Dimension functions and traces on $C^{*}$-algebras, J. Functional Analysis 45 (1982), 297-340. MR 83g:46050

5. L.G. Brown and G.K Pedersen, $C^{*}$-algebras of real rank zero, J. Func. Anal. 99 (1991), 131-149. MR 92m:46086

6. K.R. Goodearl, Von Neumann Regular Rings, Pitman, London, 1979; Second Ed., Krieger, Malabar, Fl., 1991. MR 93m:16006

7. K.R. Goodearl, Partially Ordered Abelian Groups with Interpolation, Math. Surveys and Monographs 20, Amer. Math. Soc., Providence, 1986. MR 88f:06013

8. K. C. O'Meara, Simple regular rings satisfying weak comparability, J. Algebra 141 (1991), 162-186. MR 92m:16009

9. E. Pardo, On the classification of simple Riesz groups, Preprint.

10. E. Pardo, Metric completions of ordered groups and $K_{0}$ of exchange rings, Preprint.

11. F. Wehrung, Injective positively ordered monoids I, J. Pure Appl. Algebra 83 (1992), 43-82. MR 93k:06023

12. S. Zhang, Diagonalizing projections in multiplier algebras and in matrices over a $C^{*}$-algebra, Pacific J. Math. 145 (1990), 181-200. MR 92k:46088

Departament de Matemàtiques, Universitat Autònoma de Barcelona, 08193 BellaTERRA (BARCELONA), SPAIN

E-mail address: para@mat.uab.es

E-mail address: epardo@mat.uab.es 